\title{
Lidil
}

Revue de linguistique et de didactique des langues

$64 \mid 2021$

Le passif dans la langue parlée

\section{L'acquisition des prépositions à et de en français par des locuteurs tchèques}

The Acquisition of the French Prepositions à and de by Czech speakers

\section{Hana Gruet-Skrabalova et Iva Dedková}

\section{(2) OpenEdition}

1 Journals

Édition électronique

URL : https://journals.openedition.org/lidil/9663

DOI : $10.4000 /$ lidil. 9663

ISSN : 1960-6052

Éditeur

UGA Éditions/Université Grenoble Alpes

Édition imprimée

ISBN : 978-2-37747-315-1

ISSN : $1146-6480$

Référence électronique

Hana Gruet-Skrabalova et Iva Dedková, «L'acquisition des prépositions à et de en français par des locuteurs tchèques », Lidil [En ligne], 64 | 2021, mis en ligne le 01 novembre 2021, consulté le 26 novembre 2021. URL : http://journals.openedition.org/lidil/9663 ; DOI : https://doi.org/10.4000/lidil. 9663

Ce document a été généré automatiquement le 26 novembre 2021.

(c) Lidil 


\title{
L'acquisition des prépositions à et de en français par des locuteurs tchèques
}

The Acquisition of the French Prepositions à and de by Czech speakers

\author{
Hana Gruet-Skrabalova et Iva Dedková
}

\section{Introduction}

1 Les prépositions sont des morphèmes dont le sens est essentiellement relationnel, c'est-à-dire qu'elles établissent un lien entre l'élément (mot/groupe de mots) qu'elles introduisent et un autre élément (mot) dans la phrase. Ceci est particulièrement visible avec les prépositions à et de, très fréquentes en français, qui peuvent apparaitre dans des environnements syntaxiques variés, et dont le sens varie selon le type syntaxique et le sémantisme des éléments mis en relation :

(1)

a. Marie rentre de la banque.

b. La famille semble ravie de cette nouvelle.

c. N'oublie pas d'acheter l'huile d'olive.

(2)

a. Jean habite à Lyon.

b. La population continue à lutter contre l'épidémie.

c. Il offre toujours des fleurs à sa mère.

2 La multitude des environnements et constructions dans lesquels les prépositions à et de apparaissent ainsi que la variabilité de leur sens fait que leur acquisition ou apprentissage représente une difficulté certaine pour les apprenants du français (cf. Cox, 1983 ; Gaffney, 1995).

Dans cette étude, nous nous intéressons à l'acquisition des prépositions à et de par des locuteurs tchèques, ainsi que sur le rôle positif ou négatif de la langue maternelle (LM) dans cette acquisition. Nous nous appuyons sur des travaux (Selinker, 1969, 1972; 
Kellerman \& Sharwood Smith, 1986; Odlin, 1989, 2003 ; Rutherford, 1983 ; Towell \& Hawkins, 1994) qui montrent que l'acquisition d'une langue seconde est influencée dans une certaine mesure par des propriétés lexicales et morphosyntaxiques de la LM. Nous parlerons du transfert positif lorsqu'il s'agira d'influences positives, et du transfert négatif lorsque la LM représente une source d'erreur pour l'apprenant. Nous partons de l'hypothèse qu'au cours de l'acquisition :

a. les apprenants produisent rapidement certaines constructions de manière correcte ;

b. les apprenants produisent systématiquement certaines erreurs ;

c. la source de (certaines de) ces constructions ou erreurs peut être localisée dans la LM.

4 Nous tentons ainsi de répondre aux questions suivantes :

1. Quelles constructions examinées les apprenants produisent-ils correctement ? Ce traitement correct peut-il être dû à un transfert positif?

2. Quels types d'erreurs les apprenants font-ils systématiquement ? Quelles erreurs pourraient résulter de l'influence de la LM-?

3. L'apprentissage de certaines constructions dès le début du cursus permet-il de les fixer rapidement (et définitivement), et d'éviter ainsi des erreurs?

5 L'article s'organise comme suit. Dans la section 2, nous présentons différentes constructions avec les prépositions à et de que nous avons étudiées et leurs équivalents en tchèque. Nous émettons également des hypothèses sur les possibles transferts (positifs et négatifs) du tchèque vers le français. Dans la section 3 , nous présentons l'expérience que nous avons menée auprès des locuteurs tchèques et les résultats obtenus. Enfin, la section 4 propose une discussion de ces résultats et une évaluation de nos hypothèses. Nous y répondons aux questions posées ci-dessus.

\section{Les constructions avec les prépositions à et de et les hypothèses sur leur acquisition}

\subsection{Les constructions étudiées}

Les prépositions à et de introduisent des compléments régis par différents éléments recteurs : verbe, nom, adjectif ou adverbe de quantité. Dans cette étude, nous nous sommes intéressées à ces quatre environnements syntaxiques, en distinguant plusieurs types de constructions syntaxiques, présentés de manière synthétique dans le tableau 1.

7 Lorsque l'élément recteur est un verbe, le complément introduit par la préposition correspond à l'une des catégories suivantes: pronom, syntagme nominal (SN), syntagme verbal infinitif $\left(\mathrm{SV}_{\text {inf }}\right)$. Les verbes eux-mêmes peuvent sous-catégoriser :

- un seul complément introduit par à ou de : Jean habite à Lyon. / Marie rentre de la banque ;

- deux compléments dont un introduit par à ou de : La maitresse autorise les enfants à sortir. / Jeanne empêche les voleurs de s'échapper ;

- deux compléments dont un introduit par à et l'autre par de: Paul promet à sa fille d'aller à Paris.

Lorsque l'élément recteur est un nom ou un adjectif, la préposition introduit un complément sous forme d'un nom ou d'un syntagme nominal : Marie apporte les assiettes à dessert. / La police tient le voleur de bijoux. Le directeur est favorable à mon inscription. / Elle 
a les mains noires de poussière. Par ailleurs, la préposition à peut également introduire un complément d'adjectif sous forme $\mathrm{SV}_{\text {inf }}$ : Les étudiants sont prêts à commencer les épreuves.

Enfin, les adverbes de quantité comme peu, beaucoup peuvent être suivis d'un SPrép comportant la préposition de et un nom : Beaucoup de gens ont voté pour Trump.

Tableau 1. - Constructions étudiées.

\begin{tabular}{|c|c|c|}
\hline $\begin{array}{l}\text { Élément } \\
\text { recteur }\end{array}$ & Construction & Exemple \\
\hline \multirow[t]{5}{*}{ Verbe } & $\mathrm{V}+\grave{a}+\mathrm{SN} / \mathrm{Pro} / \mathrm{SV}_{\text {inf }}$ & $\begin{array}{l}\text { Jean habite } \grave{a} \text { Lyon. } \\
\text { Marie renonce } a \grave{~ p a r t i r . ~}\end{array}$ \\
\hline & $\mathrm{V}+d e+\mathrm{SN} /$ Pro/SV $\mathrm{Snf}_{\text {f }}$ & $\begin{array}{l}\text { Marie rentre } d e \text { la banque. } \\
\text { J'envisage } d e \text { vivre en Angleterre. }\end{array}$ \\
\hline & $\mathrm{V}+\mathrm{SN} /$ Pro $+\grave{a}+\mathrm{SN} /$ Pro/SV $\mathrm{inf}$ & $\begin{array}{l}\text { Il a prêté sa moto } a \text { Caroline. } \\
\text { La maitresse autorise les enfants } a ̀ \text { sortir. }\end{array}$ \\
\hline & $\mathrm{V}+\mathrm{SN} /$ Pro $+d e+\mathrm{SN} / \mathrm{SV}_{\mathrm{inf}}$ & $\begin{array}{l}\text { Marie félicite Paul } d e \text { sa réussite. } \\
\text { Jeanne empêche les voleurs } d e \text { s'échapper. }\end{array}$ \\
\hline & $\mathrm{V}+\grave{a} \mathrm{SN} /$ Pro $+d e+\mathrm{SN} / \mathrm{SV}_{\mathrm{inf}}$ & $\begin{array}{l}\text { La vieille femme parle souvent } d e \text { sa jeunesse. } \\
\text { Paul promet } a \text { sa fille } d \text { 'aller à Paris. }\end{array}$ \\
\hline \multirow[t]{2}{*}{ Nom } & $\mathrm{N}+\grave{a}+\mathrm{N}$ & Marie apporte les assiettes $\grave{a}$ dessert. \\
\hline & $\mathrm{N}+d e+\mathrm{SN} / \mathrm{N}$ & La police tient le voleur $d e$ bijoux. \\
\hline \multirow[t]{2}{*}{ Adjectif } & $\mathrm{Adj}+\grave{a}+\mathrm{SN} / \mathrm{SV}_{\mathrm{inf}}$ & $\begin{array}{l}\text { Le directeur est favorable } a \text { mon inscription. } \\
\text { Les étudiants sont prêts } \grave{a} \text { commencer les épreuves. }\end{array}$ \\
\hline & $\mathrm{Adj}+d e+\mathrm{SN} / \mathrm{N}$ & Elle a les mains noires $d e$ poussière. \\
\hline Adverbe & $\mathrm{Adv}+d e+\mathrm{N}$ & Beaucoup de gens ont voté pour Trump. \\
\hline
\end{tabular}

Sur le plan sémantique, les prépositions à et de peuvent apparaitre dans des constructions semblables (p.ex. spatiales ou temporelles), avec une dénotation typiquement opposée (cf. Riegel et coll., 2018, p. 603). Ainsi, avec des verbes de mouvement, à dénote la direction vers un point, et de l'éloignement depuis un point. De même pour le sens temporel, avec p. ex. travailler de $9 \mathrm{~h} \grave{a}$ 17h. Avec des verbes de "transaction» (donner/recevoir), à introduit typiquement le bénéficiaire et de la source de cette transaction.

11 Dans d'autres constructions, cependant, la généralisation du sens lié à l'emploi des prépositions à ou de devient plus difficile. En effet, à peut marquer une situation spatiale (habiter à Lyon) ou temporelle (déjeuner à midi), la finalité (un verre à vin, donner sa moto à réparer), l'objet de dire, de pensée ou de croyance (penser à son avenir, croire à l'astrologie), une partie (fruit à pépins), etc. De son côté, de peut marquer l'origine (l'équipe de France), la matière (un bonbon de chocolat), la cause (avoir les mains noires de poussière), le contenu (un verre de vin), etc. Une classification détaillée de ces «sens", comme chez Marque-Pucheu(2008), montre surtout la diversité d'emplois ou d'environnements sémantiques dans lesquels ces prépositions apparaissent. Nous montrons dans la section 2.2 que seules les dénotations typiques et régulières facilitent l'acquisition des constructions syntaxiques, et fonctionnent comme des biais pour la mise en place d'un transfert positif.

\subsection{Les équivalents tchèques des prépositions à et de dans les constructions étudiées}

Le tchèque est une langue flexionnelle avec un système casuel de sept cas (nominatif, génitif, datif, accusatif, vocatif, locatif, instrumental). Le cas du nom est typiquement 
régi par le mot qui le sous-catégorise. Les verbes sous-catégorisent les compléments nominaux à qui ils assignent un cas précis, ou des compléments prépositionnels où le cas du nom est régi par la préposition. L'aspect verbal est exprimé d'une manière lexicale (sur le radical ou par les affixes) et les $\mathrm{SV}_{\text {inf }}$ (ou subordonnées infinitives) sont peu fréquentes.

De par leurs propriétés syntaxiques et sémantiques, les prépositions qui nous intéressent ici peuvent avoir plusieurs équivalents en tchèque : prépositionnel, casuel, verbal, conjonctif ou aucun. Nous verrons que certains équivalents sont réguliers et donc prévisibles, d'autres, ayant un sens abstrait ou étant plus idiosyncratiques, sont non prévisibles.

\subsection{La préposition à}

Voici les équivalents possibles de la préposition française à :

\section{a) Équivalents prépositionnels}

Dans les constructions verbales spatiales, la préposition à dénote la direction vers un point ou une situation spatiale. Elle correspond régulièrement à la préposition tchèque $v$ («dans », sens statique) lorsque le verbe se combine avec un complément de lieu statique, et à la préposition do (sens directionnel), lorsque le verbe se combine avec un complément de lieu directionnel. Ces constructions sont par ailleurs présentes très tôt dans les manuels du FLE.

(3)

a. Jean habite à Lyon. > Jean bydlí $v$ Lyonu.

b. Jean va à Lyon. > Jean jede do Lyonu.

Une troisième préposition, na («sur»), fonctionne comme équivalent de à dans la construction « $\mathrm{V}$ à $\mathrm{N}$ » avec des verbes psychologiques ou de dire (cf. 4a), et dans la construction « $\mathrm{N}$ à $\mathrm{N}$ », lorsque le deuxième $\mathrm{N}$ précise le sens du premier (cf. $4 \mathrm{~b}$ ). Dans ce dernier cas, la séquence "à $\mathrm{N}$ » caractérisant le premier $\mathrm{N}$ peut également être exprimée par une modification adjectivale. La préposition na peut être problématique pour les constructions " $\mathrm{V}$ à $\mathrm{N}$ » en français, car son équivalent locatif concret "sur " (sur la table > na stole) est habituellement présenté aux apprenants avant son sens abstrait.

(4)

a. Il pense à son avenir > Myslí na svou budoucnost.

b. une assiette à desser > talî́rek na moučnik / dezertní talírek

D'autres prépositions moins prévisibles peuvent également répondre à la préposition à, cf. od (« de », « depuis », sens abstrait), s (« avec ») et $v$ (« dans », sens abstrait) en (5). Nous verrons dans la section 2.2.2 que les prépositions od et $s$ sont aussi des équivalents possibles de la préposition de, ce qui peut constituer une source d'erreur dans les exemples comme (5a).

(5)

a. Pierre a emprunté le vélo à son frère. > Pavel si vypủjčil kolo od svého bratra.

b. Marie croit à l'astrologie. > Marie věř́ v astrologii.

c. des fruits à pépins > ovoce s peckami

\section{b) Équivalents casuels}

Dans les constructions verbales, le cas datif est un équivalent régulier de à lorsque le verbe se combine avec un complément bénéficiaire. Il s'agit souvent de verbes à double 
objet, comme en (6a). Les verbes bi-transitifs comme donner ou offrir qch à qqn sont abordés par ailleurs très tôt dans les manuels de FLE.

(6)

a. Je dois deux euros à Marie. > Dlužím dvě eura Marii (N-dat).

b. Ce sac appartient à ma cousine. > Ta taška patř̌ mé sestřenici (N-dat).

19 Avec d'autres verbes, dont le complément n'est pas un bénéficiaire (mais p. ex. une source), le complément introduit en français par à sera en tchèque au cas génitif ou accusatif. Le cas morphologique permet même de désambigüiser un verbe, comme pour demander : le complément au génitif exprimera la personne à qui on pose une question (demander > zeptat se), alors que le complément à l'accusatif exprimera la personne à qui on présente une demande (demander > požádat) :

7)

a. Demande à ta mère quelle heure il est. > Zeptej se maminky (N-gen), kolikje hodin.

b. Demande à ta mère de s'occuper. > Požádej maminku (N-acc), aby se ti postarala o kocoura.

\section{c) Autres équivalents}

Dans les constructions de type " $\mathrm{V}$ à $\mathrm{SV}_{\text {inf }}$ " et après les adjectifs, la préposition à n'a pas d'équivalent prévisible. Le tchèque utilise ici un verbe infinitif nu (cf. 8a), un substantif déverbal introduit par la préposition na («sur») (cf.8b), ou une subordonnée complétive introduite par aby ou že (cf. 8c). Ces mêmes équivalents sont possibles avec la préposition de (cf. section 2.2.3).

8)

a. Paul a donné sa moto à réparer. > Pavel si dal ø opravit motorku.

b. Les étudiants sont prêts à commencer les épreuves. > Studenti jsou připraveni na

zkoušení.

c. La maitresse autorise les enfants à sortir. > Učitelka dovolila dětem, aby odešly.

\subsection{La préposition de}

La préposition française de correspond aux mêmes types d'équivalents que la préposition à :

\section{a) Équivalents prépositionnels}

Dans les constructions verbales spatiales, où de dénote l'éloignement d'un lieu, de correspond régulièrement à la préposition $z$ («de», «depuis», sens spatial). La préposition $o$ est un équivalent typique de de dans les constructions où le verbe se combine avec un complément désignant le thème. L'apprentissage de ces deux constructions intervient dès les premières étapes du parcours FLE, lors de l'introduction des verbes de mouvement (revenir de > vrátit se z) ou de dire (parler de > mluvit o) (cf. 9ab). Avec les verbes à deux compléments, la préposition de introduisant la source correspond en tchèque à la préposition od.

(9)

a. Marie rentre de la banque. > Marie se vrací z banky.

b. Marie s'occupe de sa fille. > Marie se stará o svou dceru.

c. Elle a reçu un cadeau de son fiancé. > Dostala dárek od svého snoubence.

D'autres prépositions tchèques apparaissent dans les constructions verbales et adjectivales, mais leur emploi ne peut être facilement associé à un aspect sémantique et est donc moins prévisible : od et $z$ (« de », sens causal), s (« avec »), $k$ (« vers », « chez »), do (« dans » et « à » directionnels). 
(10)

a. Elle a les mains noires de poussière. > Má ruce černé od prachu.

b. Elle est ravie de cette nouvelle. > Je nadšená z té zprávy.

c. Maman est contente de notre travail. > Maminka je spokojená s naší prací.

d. Marie félicite Paul de sa réussite. > Marie blahopřeje Pavlovi $k$ úspěchu.

e. La reine est tombée amoureuse de son valet.> Královna se zamilovala do svého

komorného.

b) Équivalents casuels

Après les adverbes de quantité ou dans la construction « $\mathrm{N}$ de $\mathrm{N}$ » exprimant une relation d'appartenance, la préposition de correspond toujours au cas génitif. Ces constructions sont introduites dans les manuels du FLE dès les premières leçons.

(11)

a. beaucoup de gens $>$ mnoho lidí (N-gen)

b. le voleur de bijoux > zloděj šperků (N-gen)

Dans la construction « $\mathrm{N}$ de $\mathrm{N}$ », le deuxième $\mathrm{N}$ peut également caractériser le premier : cette relation peut être exprimée en tchèque par un adjectif qualitatif ou un adjectif possessif.

(12)

a. l'huile d'olive > olivový olej (Adj)

b. la cave de mon grand-père > dědečkův sklep (Adj possessif)

Dans les constructions verbales, le complément introduit par de peut devenir un syntagme nominal au cas régi par le verbe, p. ex. instrumental ou accusatif.

(13)

a. menacer (qqn) de mort > vyhrožovat (někomu-dat) smrtí (N-instr)

b. se servir de son bâton > používat svou hưl ( $\mathrm{N}-\mathrm{acc})$

\section{c) Autres équivalents}

Lorsque les verbes et les adjectifs se combinent avec un $\mathrm{SV}_{\text {inf }}$ l'équivalent fréquent de de est une subordonnée complétive introduite par la conjonction že (que + indicatif) ou aby (que + conditionnel) (cf. 14). Par ailleurs, dans de nombreuses constructions «V de $\mathrm{V}$ ", aucun élément particulier ne relie explicitement les verbes reliés par de en français. Comme pour la préposition à, le tchèque peut utiliser dans ces cas un verbe infinitif nu (cf. 15a), ou un substantif déverbal introduit par la préposition od (cf. 15b).

(14)

a. Elle suggère à son mari de partir. > Navrhuje manželovi, aby odjel(i).

b. J'envisage de vivre en Angleterre. > Plánuji, že budu žít v Anglii.

(15)

a. N'oublie pas d'acheter de l'huile d'olive. > Nezapomeň koupit olivový olej.

b. Marie m'a découragé de continuer le chant. > Marie mě odradila od zpěvu / zpívání.

\section{Des hypothèses sur les possibles transferts}

Selon Odlin (2003), l'influence translinguistique exige une identification interlingue (angl. interlingual identification) et peut donc avoir lieu lorsque la construction de la langue cible correspond systématiquement à une construction dans la langue maternelle. Nous pouvons ainsi faire l'hypothèse que lorsque la construction prépositionnelle en français correspond systématiquement à une construction de la langue tchèque, les locuteurs pourront effectuer un transfert positif de cette construction, en s'appuyant sur une identification lexico-sémantique et morphosyntaxique. En considérant les équivalents de à et de exposés dans la section 
précédente, le transfert positif semble possible dans les cas suivants, où nous nous attendons par conséquent à peu d'erreurs :

a. cas génitif (de quantité et d'appartenance) dans les constructions « $\mathrm{N}$ de $\mathrm{N}$ » et « Adv de $\mathrm{N}$ » (la cave de mon grand-père, beaucoup de gens);

b. datif dans le contexte verbal avec un bénéficiaire introduit par à (donner à) ;

c. préposition $z$ dans le contexte verbal spatial avec de (rentrer de) ;

d. préposition $v$ dans le contexte verbal spatial avec $\grave{a}$ (habiter à).

L'acquisition de ces constructions peut être en outre facilitée par le fait qu'elles interviennent dans le parcours des apprenants dès les premières étapes. Ceci est également vrai pour les unités lexicales complexes telles que numéro de téléphone ou pêche à la ligne. De même, certains verbes figurent dans le vocabulaire de base dans la plupart des manuels (cf. Tibert, 2003 ; Mérieux \& Loiseau, 2004 ; Pravdová \& Pravda, 2007 ; Girardet \& Pécheur, 2012), ce qui suggère que les constructions comportant ces verbes pourraient être fixées dès le début de l'acquisition / apprentissage. Il s'agit de verbes comme :

- donner qch à qqn (dát N-acc N-dat) ;

- dire qch à qqn (ř́ci N-acc N-dat) ;

- demander qch à qqn (zeptat se N-gen na N-acc / požádat N-acc o N-acc);

- aider qqn à faire qch (pomoci N-dat udèlat N-acc / pomoci N-dat s N-instr);

- réussir à faire qch (SN-dat V pronominal 'podariit se' $S V_{\text {inf }}$ ).

D'autres verbes peuvent être plus difficiles à acquérir, car ils sont polysémiques et apparaissent dans des constructions syntaxiques différentes, comme :

- tenir qch (držet N-acc) / tenir qch de qqn (mít N-acc po N-loc) / tenir à qch (záležet na N-loc);

- croire qqn (věřit N-dat) / croire à qch ou en qch (věrit v N-acc / N-dat).

31 À contrario, lorsque les prépositions à et de ne correspondent pas, dans la LM, à une construction prédictible et régulière (i.e. prépositions diverses, préposition zéro, propositions subordonnées), l'identification lexicale ou structurelle semble difficile, voire impossible. Il s'agit notamment de constructions «V à / de V » ou «Adj à V ", dans lesquelles nous pouvons nous attendre à beaucoup d'erreurs.

Nous émettons trois hypothèses de transfert relatives à ces constructions :

a. La préposition de étant plus fréquente devant un $\mathrm{SV}_{\text {inf }}$ (cf. Huot, 1981 ; Lonsdale \& Le Bras, 2011), sa surgénéralisation parait plausible dans ces contextes. L'importance de la fréquence d'un item lexical dans l'input pour son acquisition a été démontrée par De Groot et Keizjer (2000) ou Kirjavainen et coll. (2009).

b. Dans les constructions verbales avec deux compléments, le complément bénéficiaire au datif est typiquement associé à la préposition à, et le complément source introduit par la préposition od à la préposition de. Nous pouvons ainsi nous attendre à un transfert négatif dans les cas comme emprunter qch à qqn. Ce transfert est d'autant plus plausible que le tchèque utilise un seul verbe (sous forme non pronominale vs pronominale) pour les verbes prêter qch à qqn (půjčit N-acc N-dat) et emprunter qch à qqn (půjčit si N-acc od N-gen).

c. Les prépositions ayant un équivalent unique concret peuvent également donner lieu à un transfert négatif. Ainsi nous pouvons nous attendre à un transfert négatif dans le cas de la préposition $s$ («avec») dans la construction adjectivale spokojený $s$ něčím, littéralement 'content avec quelque chose', au lieu de content de quelque chose. 


\section{La tâche expérimentale} expérimentale comportant deux tests. Nous décrirons ensuite ces tests et expliquerons la méthodologie utilisée pour leur évaluation. Enfin, nous exposerons les résultats obtenus.

\subsection{Le profil des sujets}

Nos tests s'adressaient à 52 locuteurs natifs de tchèque inscrits en mention Philologie française au département d'études romanes de l'Université d'Ostrava et ont eu lieu au printemps 2017. Les étudiants devaient d'abord répondre à un questionnaire qui consistait à indiquer leur âge, sexe et langue maternelle, le niveau de leurs études (Licence ou Master), la discipline étudiée, la durée d'apprentissage du français et le niveau de français (évaluation subjective). Voici le profil des sujets interrogés :

- Sexe : 44 femmes et 8 hommes.

- Âge : 39 sujets âgés de 19 à 24 ans ; 9 sujets de 25 à 30 ans ; 4 sujets de 31 ans et plus.

- Niveau d'études : 38 sujets en Licence (dont 17 en L1, 16 en L2 et 5 en L3) et 14 sujets en Master (6 en M1 et 8 en M2).

- Disciplines étudiées: Français dans le domaine de l'entreprise (L), Langue et littérature françaises - études bivalentes ${ }^{2}(\mathrm{~L})$, Français pour la traduction(M), Enseignement du français dans le secondaire - études bivalentes (M).

- Durée d'apprentissage du français : entre 2 et 16 ans (cf. tableau 2).

- Niveau de français (évaluation subjective) : entre B1 et C1 (cf. tableau 3).

Étant donné que l'évaluation du niveau de français ne s'appuyait pas sur un certificat en langue, nous avons préféré diviser les sujets en deux groupes selon la durée d'apprentissage du français ${ }^{3}$ :

- Groupe $1: 34$ sujets (2 à 8 ans d'apprentissage) ;

- Groupe $2: 18$ sujets (9 à 16 ans d'apprentissage).

Tableau 2. - Durée d'apprentissage du français.

\begin{tabular}{|l|c|c|c|}
\hline Nb d'années & $2-5$ & $6-8$ & $9-16$ \\
\hline Nb de sujets & 8 & 25 & 18 \\
\hline
\end{tabular}

Tableau 3. - Niveau de français - évaluation subjective.

\begin{tabular}{|l|c|c|c|c|}
\hline Niveau & B1 & B2 & B2-C1 & C1 \\
\hline Nb de sujets & 25 & 14 & 2 & 10 \\
\hline
\end{tabular}

Par ailleurs, les sujets avaient des connaissances en d'autres langues étrangères (cf. tableau 4), mais avec le niveau B2-C2 seulement en anglais. Bien que ces connaissances aient pu avoir une influence sur leur compétence en français, nous n'avons pas pu 
aborder cette question dans le cadre de notre étude en raison de la longueur limitée de l'article.

Tableau 4. - Autres langues parlées/étudiées.

\begin{tabular}{|l|c|c|c|c|c|c|c|c|}
\hline Langue & Anglais & Espagnol & Allemand & Italien & Russe & Chinois & Polonais & Suédois \\
\hline Nb de sujets & 44 & 16 & 20 & 7 & 4 & 2 & 1 & 1 \\
\hline
\end{tabular}

\subsection{Les tests}

Les tests que nous avons construits et soumis à nos sujets avaient pour objectifs de déterminer :

a. quelles sont les constructions que les apprenants produisent correctement ou incorrectement ;

b. si ce traitement (in)correct peut être dû à un transfert (positif ou négatif) à partir de la LM ;

c. si l'apprentissage de certaines constructions dès le début du cursus aide à les fixer rapidement, et à éviter ainsi certaines erreurs.

\subsubsection{La description des tests}

Le premier test comportait 50 phrases grammaticales et agrammaticales, dont 24 concernaient la préposition à, 23 la préposition de (présente deux fois dans une des phrases), 1 la préposition à ou de et 3 les deux prépositions (constructions avec deux compléments verbaux). Les sujets devaient décider si ces phrases étaient grammaticales $(\mathrm{G})$ ou agrammaticales $(\mathrm{AG})$, puis proposer une correction pour les phrases jugées agrammaticales.

Le deuxième test comportait 49 phrases à trous, que les sujets devaient compléter par une préposition ou par rien (-). 14 de ces phrases ciblaient la préposition à, 20 la préposition de, 4 pouvaient contenir l'une ou l'autre et 11 étaient correctes sans préposition pour éviter que les apprenants "repèrent » les prépositions testées et focalisent leur attention uniquement sur elles.

Pour construire ces tests, nous nous sommes appuyées sur des manuels utilisés dans l'enseignement du FLE en République tchèque ${ }^{4}$. Le vocabulaire employé était donc censé être connu des sujets interrogés.

\subsubsection{L'évaluation des tests}

41 Avant de présenter les résultats des tests, il convient d'apporter quelques remarques sur la méthodologie de leur évaluation et la notion des réponses "correcte » et « incorrecte », notamment dans le test 1 (jugement de grammaticalité).

Le test de grammaticalité comportait deux types de phrases: grammaticales $(\mathrm{G})$ et agrammaticales $(\mathrm{AG})$. Nous avons considéré deux types de réponses comme correctes :

a. lorsqu'une phrase $\mathrm{G}$ a été jugée grammaticale, $\mathrm{p}$. ex. Le président revient de Pékin ;

b. lorsqu'une phrase AG a été jugée agrammaticale et corrigée correctement, p. ex. *Il pense de son avenir. $\rightarrow$ Il pense à son avenir. 
À contrario, nous avons considéré comme réponse incorrecte les cas suivants :

a. lorsqu'une phrase $\mathrm{G}$ a été jugée agrammaticale à cause de la préposition employée et que la correction proposait une autre préposition, même si celle-ci pouvait être correcte, p. ex. Le président revient de Pékin $\rightarrow$ Le président revient à Pékin. La réponse montre en effet que les sujets n'ont pas acquis la construction testée revenir de Pékin;

b. lorsqu'une phrase AG a été jugée agrammaticale, mais corrigée incorrectement, p.ex. ${ }^{*}$ Tu nous aides - terminer ce déménagement $\rightarrow{ }^{*}$ Tu nous aides de terminer ce déménagement.

Par ailleurs, l'emploi des prépositions à et de est inséparablement lié à la problématique des déterminants. Lorsqu'il s'agit des constructions présentées aux apprenants dès le début du cursus, seules les formes correctes sont comptabilisées dans les réponses correctes, p. ex. un verre du vin au lieu de un verre de vin était considéré comme une réponse incorrecte. À l'inverse, dans les cas plus difficiles, abordés plus tard dans le cursus, même les formes fautives sont comptabilisées dans les réponses correctes, à condition que la bonne préposition (à ou de) soit utilisée, p. ex. aux pépins dans Nous avons goûté des fruits à pépins ou * de la Normandie dans Charlotte revient de Normandie.

Enfin, faute d'espace, nous n'avons pas inclus dans la présente étude les phrases sans préposition, dans lesquelles les sujets ont proposé d'utiliser de ou à, même si cela aurait pu être éclairant.

\subsection{Les résultats des tests}

Cette section résume les résultats de nos tests. Nous nous intéressons aux paramètres suivants liés à l'utilisation correcte des prépositions à et de :

- niveau des sujets (G1 vs G2) ;

- type de test ;

- phrases grammaticales vs agrammaticales ;

- type d'élément recteur ;

- type de complément avec l'élément recteur verbal et adjectival.

47 a) Nous constatons tout d'abord que tous les résultats obtenus sont meilleurs pour le G2, qu'il s'agisse du test 1 ou 2, et de la préposition à ou de. Le G2 obtient en effet des résultats d'environ $10 \%$ plus élevés que le G1 pour les deux tests. La durée de l'apprentissage améliore donc le taux de réussite, comme nous pouvions nous y attendre (cf. tableau 5).

Comparant l'emploi des prépositions à et de (cf. tableau 5 également), nous constatons que les étudiants des deux groupes obtiennent, dans les deux tests, de meilleurs résultats dans les constructions avec la préposition de. Ceci confirme l'hypothèse que de serait acquis plus rapidement, l'hypothèse liée à la fréquence plus importante de la préposition de dans l'input et donc de sa possible surgénéralisation.

Tableau 5. - Réponses correctes avec à et de selon le test et le niveau des sujets (G1/G2) 5 .

\begin{tabular}{|l|ll|ll|cl|cl|}
\hline \multirow{2}{*}{ Préposition } & \multicolumn{2}{|l|}{ Test 1 Réponses correctes } & \multicolumn{3}{l|}{ Test 2 : Réponses correctes } \\
\cline { 2 - 9 } & \multicolumn{2}{|c|}{ G1 } & \multicolumn{2}{c|}{ G2 } & G1 & \multicolumn{2}{c|}{ G2 } \\
\hline$\grave{a}$ & $503 / 918$ & $55 \%$ & $319 / 486$ & $66 \%$ & $228 / 476$ & $48 \%$ & $141 / 252$ & $56 \%$ \\
\hline$d e$ & $653 / 915$ & $71 \%$ & $384 / 483$ & $80 \%$ & $482 / 680$ & $71 \%$ & $289 / 360$ & $80 \%$ \\
\hline$\grave{a}$ ou $d e$ & $15 / 34$ & $44 \%$ & $13 / 18$ & $72 \%$ & $116 / 136$ & $85 \%$ & $70 / 72$ & $97 \%$ \\
\hline
\end{tabular}


49 b) Nous constatons par ailleurs que les phrases grammaticales dans le test de jugement (cf. tableau 6) ont obtenu significativement plus de réponses correctes que les phrases agrammaticales. Ceci peut être dû au fait que le traitement des phrases agrammaticales demande un effort cognitif plus important. Une autre explication peut résider dans la subtilité des structures proposées dans les phrases agrammaticales. Par exemple, en comparant les réponses du G1 aux deux phrases avec la structure "Adj à SV $\mathrm{SV}_{\text {inf }}$, nous notons 30 réponses correctes pour la phrase Cet achat était indispensable à faire et seulement 10 réponses correctes pour la phrase *Le français n'est pas facile d'apprendre. Cependant, en fonction du sujet, l'adjectif facile peut être suivi d'un complément à $S V_{\text {inf }}$ ou à $S V_{\text {inf }}$ : Le français n'est pas facile à apprendre vs Il n'est pas facile d'apprendre le français, ce qui pourrait expliquer l'acceptabilité de la phrase *Lefrançais n'est pas facile d'apprendre pour les apprenants. De même, en comparant deux phrases comportant le verbe donner, nous notons que la phrase Le professeur a donné un devoir aux élèves a obtenu seulement 2 réponses incorrectes, tandis que la phrase *Jean a donné une fleur de Suzanne, que nous considérons comme agrammaticale car elle est syntaxiquement et sémantiquement incomplète hors contexte, en a obtenu 7. Cependant, cette dernière peut devenir acceptable si le contexte permet de reconstituer le bénéficiaire de la transaction de donner.

Tableau 6. - Test 1 : réponses correctes aux phrases G et AG.

\begin{tabular}{|l|ll|cc|ll|ll|}
\hline \multirow{2}{*}{ Préposition } & \multicolumn{4}{|c|}{ Réponses correctes aux phrases G } & \multicolumn{3}{c|}{ Réponses correctes aux phrases AG } \\
\cline { 2 - 9 } & \multicolumn{2}{|c|}{ G1 } & \multicolumn{2}{c|}{ G2 } & \multicolumn{2}{c|}{ G1 } & \multicolumn{2}{c|}{ G2 } \\
\hline$\grave{a}$ & $358 / 442$ & $81 \%$ & $203 / 234$ & $87 \%$ & $145 / 476$ & $30 \%$ & $116 / 252$ & $46 \%$ \\
\hline$d e$ & $558 / 680$ & $82 \%$ & $320 / 360$ & $89 \%$ & $98 / 238$ & $41 \%$ & $67 / 126$ & $53 \%$ \\
\hline$\grave{a}+d e$ & $916 / 1122$ & $82 \%$ & $523 / 594$ & $88 \%$ & $243 / 714$ & $34 \%$ & $183 / 378$ & $48 \%$ \\
\hline
\end{tabular}

50 c) Les résultats diffèrent également en fonction des environnements et des constructions étudiés (cf. tableau 7).

51 Nous notons de très bons résultats pour la construction « $\mathrm{Adv}$ de $\mathrm{N}$ » dans les deux tests : en moyenne $82,5 \%$ (G1) et 95,5\% (G2) des réponses correctes. Ces résultats peuvent s'expliquer, d'une part, par le transfert positif (cf. section 2.3), et, d'autre part, par le fait que l'apprentissage de cette construction intervient dès les premières étapes du parcours d'apprentissage du FLE.

52 Nous constatons également une différence importante entre les constructions « $\mathrm{N}$ de $N$ » et « $N$ à $N$ », en particulier dans le test 2 , avec $68,5 \%$ (G1) et $76 \%$ (G2) des réponses correctes pour « $\mathrm{N}$ de $\mathrm{N}$ » (un verre de vin) vs $30 \%$ (G1) / $44 \%$ (G2) pour « $\mathrm{N}$ à $\mathrm{N} »$ (un verre à vin). Cette différence pourrait être attribuée notamment au transfert positif de la première construction, et à la non-existence d'un équivalent régulier pour la deuxième. Pourtant, certaines constructions comme un verre à vin ou une glace à la fraise sont présentes très tôt dans les manuels de FLE.

53 De même, de meilleurs résultats peuvent être observés pour la préposition de dans la construction « Adj de N » vs « Adj à N ».

54 À contrario, il n'y a pas de différences significatives entre les différentes constructions dépendant d'un verbe: nous constatons en moyenne $62 \%$ (G1) / 72,5\% (G2) des réponses correctes. 
Tableau 7. - Réponses correctes en fonction des environnements et des constructions étudiés.

\begin{tabular}{|c|c|c|c|c|c|c|c|c|c|}
\hline \multirow{2}{*}{\multicolumn{2}{|c|}{ Élément recteur }} & \multicolumn{4}{|c|}{$\begin{array}{l}\text { Test 1: } \\
\text { Réponses correctes }\end{array}$} & \multicolumn{4}{|c|}{$\begin{array}{l}\text { Test 2: } \\
\text { Réponses correctes }\end{array}$} \\
\hline & & \multicolumn{2}{|c|}{$\mathrm{G} 1$} & \multicolumn{2}{|c|}{ G2 } & \multicolumn{2}{|c|}{ G1 } & \multicolumn{2}{|c|}{ G2 } \\
\hline \multirow[t]{6}{*}{ Verbe } & $\mathrm{V} \grave{a} \ldots$ & $149 / 272$ & $55 \%$ & $93 / 144$ & $65 \%$ & $61 / 93$ & $66 \%$ & $38 / 51$ & $75 \%$ \\
\hline & $\mathrm{V} d e \ldots$ & $265 / 371$ & $71 \%$ & $155 / 195$ & $79 \%$ & $87 / 152$ & $57 \%$ & $60 / 75$ & $80 \%$ \\
\hline & V SN $a ̀ \ldots$ & $180 / 238$ & $76 \%$ & $112 / 126$ & $89 \%$ & $135 / 236$ & $57 \%$ & $90 / 135$ & $67 \%$ \\
\hline & $\mathrm{V} \mathrm{SN} d e \ldots$ & $127 / 204$ & $62 \%$ & $76 / 108$ & $70 \%$ & $63 / 102$ & $62 \%$ & $35 / 54$ & $65 \%$ \\
\hline & $\mathrm{V} a ̀ \mathrm{SN} d e \ldots$ & $30 / 102$ & $29 \%$ & $27 / 54$ & $50 \%$ & $95 / 136$ & $70 \%$ & $53 / 72$ & $74 \%$ \\
\hline & Total & $751 / 1187$ & $63 \%$ & $463 / 627$ & $74 \%$ & $441 / 719$ & $61 \%$ & $276 / 387$ & $71 \%$ \\
\hline \multirow[t]{3}{*}{ Nom } & 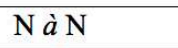 & $58 / 189$ & $31 \%$ & $40 / 95$ & $42 \%$ & $27 / 93$ & $29 \%$ & $24 / 52$ & $46 \%$ \\
\hline & $\mathrm{N} d e \mathrm{~N}$ & $52 / 102$ & $51 \%$ & $34 / 54$ & $63 \%$ & $167 / 194$ & $86 \%$ & $76 / 58$ & $89 \%$ \\
\hline & Total & $110 / 291$ & $38 \%$ & $74 / 149$ & $50 \%$ & $194 / 287$ & $68 \%$ & $100 / 137$ & $73 \%$ \\
\hline \multirow[t]{3}{*}{ Adjectif } & $\operatorname{Adj} \grave{a} \ldots$ & $74 / 136$ & $54 \%$ & $43 / 72$ & $60 \%$ & $67 / 136$ & $49 \%$ & $45 / 72$ & $63 \%$ \\
\hline & $\operatorname{Adj} d e \ldots$ & $77 / 102$ & $75 \%$ & $49 / 54$ & $91 \%$ & $64 / 102$ & $63 \%$ & $41 / 54$ & $76 \%$ \\
\hline & Total & $151 / 238$ & $63 \%$ & $92 / 126$ & $73 \%$ & $131 / 238$ & $55 \%$ & $86 / 126$ & $68 \%$ \\
\hline Adverbe & Adv de $\mathrm{N}$ & $57 / 68$ & $84 \%$ & $34 / 36$ & $94 \%$ & $55 / 68$ & $81 \%$ & $35 / 36$ & $97 \%$ \\
\hline
\end{tabular}

d) Enfin, la nature nominale ou verbale du complément semble jouer un rôle dans l'utilisation correcte de la préposition à, contrairement à la préposition de (cf. tableau 8). Les résultats corrects les plus faibles apparaissent lorsque à est suivi d'un $S V_{\text {inf }}$ : en moyenne $45,5 \%$ (G1) et 58,5\% (G2). Il s'agit ici d'un contexte imprédictible bien que certaines des constructions testées interviennent dès les premières étapes du parcours FLE, comme aider à $S V_{\text {inf }}$ (Jean aide Marie à réparer sa voiture. > Jan pomáhá Marii opravit auto.).

Tableau 8. - Réponses correctes en fonction du type de complément (S)N/SV ${ }_{\text {inf }}$ après $V$ et Adj.

\begin{tabular}{|l|ll|ll|rl|ll|}
\hline \multirow{2}{*}{$\begin{array}{l}\text { V/Adj + Prép } \\
+ \text { complément }\end{array}$} & \multicolumn{2}{|l|}{ Test 1 : Réponses correctes } & \multicolumn{3}{l|}{ Test 2 : Réponses correctes } \\
\cline { 2 - 9 } & \multicolumn{2}{|c|}{$\mathrm{G} 1$} & \multicolumn{2}{|c|}{$\mathrm{G} 2$} & $\mathrm{G} 1$ & \multicolumn{2}{c|}{ G2 } \\
\hline$\grave{a}+(\mathrm{S}) \mathrm{N}$ & $344 / 563$ & $61 \%$ & $212 / 293$ & $72 \%$ & $135 / 202$ & $67 \%$ & $83 / 117$ & $71 \%$ \\
\hline$d e+(\mathrm{S}) \mathrm{N}$ & $268 / 371$ & $72 \%$ & $158 / 195$ & $81 \%$ & $184 / 274$ & $67 \%$ & $98 / 135$ & $73 \%$ \\
\hline$\grave{a}+\mathrm{SV}_{\text {inf }}$ & $101 / 238$ & $42 \%$ & $67 / 126$ & $53 \%$ & $128 / 263$ & $49 \%$ & $90 / 141$ & $64 \%$ \\
\hline$d e+\mathrm{SV}_{\text {inf }}$ & $306 / 442$ & $69 \%$ & $184 / 234$ & $79 \%$ & $161 / 254$ & $63 \%$ & $100 / 129$ & $78 \%$ \\
\hline
\end{tabular}

\section{Discussion des résultats}

Les résultats présentés dans la section précédente permettent d'apporter des arguments en faveur des trois hypothèses énoncées dans la section 2. Les sujets obtiennent en effet de très bons résultats dans les constructions où un transfert positif est envisageable en raison d'une équivalence lexicale ou syntaxique régulière. Il s'agit des constructions suivantes :

- $z$ spatial $=$ de spatial $:$ Prezident se vrací $z$ Pekingu. $>$ Le président revient de Pékin.

- $v$ spatial $=$ à spatial $:$ Jean bydlí $v$ Lyonu. $>$ Jean habite à Lyon.

- génitif d'appartenance = de $\mathrm{N}:$ málo drog $>$ peu de drogue, sklenička vína $>$ un verre de vin.

- datif bénéficiaire = à $\mathrm{N}$ : Jan dal Zuzaně květinu. > Jean a donné une fleur à Suzanne.

- od N-gen = de $\mathrm{N}$ source $:$ Charlotte dostává dárek od snoubence. $>$ Charlotte reçoit un cadeau de son fiancé. 
- $\mathrm{V}$ o $\mathrm{N}=\mathrm{V}$ de $\mathrm{N}$ : Stará žena často mluví o svém mládí. > La vieille femme parle souvent de sa jeunesse.

- unités lexicales complexes : rybolov > la pêche à la ligne, telefonní čísla > numéros de téléphone.

A inverse, beaucoup d'erreurs apparaissent dans les constructions imprédictibles ou irrégulières, notamment avec la préposition à. La préposition à est omise (ce qui peut aussi découler d'un transfert négatif de la construction directe en tchèque) :

- $\mathrm{V} \grave{a} \mathrm{~N}>* \mathrm{~V} \mathrm{~N}$ :

*Paul désobéit souvent ses parents.

*Demande ta mère de s'occuper de ton chat.

ou remplacée par une autre préposition, typiquement de, ce qui confirme la surgénéralisation de cette dernière :

- $\mathrm{V}$ à $\mathrm{V}>*^{*} \mathrm{~V}$ de $\mathrm{V}$ :

*Je vous encourage de poursuivre vos efforts.

- Adj à $\mathrm{N}>$ *Adj de $\mathrm{N}$ :

*Le directeur est favorable de mon inscription.

- $\mathrm{N}$ à $\mathrm{N}>$ * $\mathrm{N}$ de / en $\mathrm{N}$ : *la cave de vin, *randonnée de / en cheval.

transfert négatif semble avoir lieu lorsque la construction tchèque contient une préposition ayant un équivalent concret prototypique (na stole > sur la table, stebou > avec toi) ou lorsque la construction tchèque contient un adjectif correspondant typiquement à « de $\mathrm{N}$ » (olivový olej > huile d'olive) :

- na > sur: Myslí na svou budoucnost. > *Il pense sur son avenir.

- s(e) > avec : spokojený se svou prací > *content avec son travail

- Adj $\mathrm{N}>$ de $\mathrm{N}$ : jahodová zmrzlina > *une glace de la fraise

ailleurs, lorsqu'une construction nominale admet les deux prépositions, à désignant une finalité et de un contenu, nous notons une préférence nette pour la préposition de: tous les sujets ont en effet utilisé la préposition de dans la phrase Jean prend un verre de / à vin. Cette préférence pourrait être liée à l'acquisition rapide de la construction « $\mathrm{N}$ de $\mathrm{N}$ » grâce au transfert positif du génitif d'appartenance.

La phrase Pierre a emprunté le vélo à son frère représente un cas particulier. De nombreux sujets ont utilisé ici la préposition de: Pierre a emprunté le vélo de son frère. Ce résultat peut être interprété de deux manières: il peut s'agir soit d'un transfert négatif d'un $\mathrm{N}$ source introduit en tchèque par la préposition od > de (půjčit si něco od svého bratra > *emprunter qch de son frère), soit d'un transfert positif du génitif d'appartenance vers la construction « $\mathrm{N}$ de $\mathrm{N}$ » (půjčit si N svého bratra-gen > emprunter $\mathrm{N}$ de son frère).

61 Enfin, nous avons émis l'hypothèse (cf. 2.3) que l'introduction de certaines structures dès le début du cursus pourrait faciliter leur fixation, p. ex. la voiture de Marie, donner qch à qqn ou continuer à faire qch. Ceci n'est cependant pas toujours le cas: la construction dire à qqn recueille un nombre considérable de résultats erronés (*dire qqn), ce qui est d'autant plus surprenant que le complément «à $\mathrm{N}$ » est un bénéficiaire correspondant typiquement au cas datif en tchèque. Par ailleurs, la fixation de certaines constructions semble avoir un impact négatif sur l'acquisition des constructions différentes avec le même verbe. Ainsi, le fait que les manuels de FLE indiquent que le verbe continuer se construit avec la préposition à pourrait expliquer que les sujets ont utilisé majoritairement à dans la phrase La population continue à / de lutter contre l'épidémie. De même, l'introduction de la construction servir à $V_{\text {inf }}$ avant la construction pronominale se servir de qch pourrait expliquer l'emploi de la préposition à dans la phrase Il se sert de son bâton comme arme. La fixation d'une règle peut également 
mener vers sa surgénéralisation. Ainsi, l'expression de la conséquence par la préposition pour suivie de l'infinitif en français correspond en tchèque à la proposition subordonnée de la conséquence introduite par la conjonction aby (elle fait du vélo pour être en forme > jezdí na kole, aby byla ve formě). Dans notre étude, la surgénéralisation de cette règle peut être observée dans la phrase *Jel'ai convaincu pour rester diner. < Přesvědčil jsem ho, aby zůstal na večeři.

\section{Conclusion}

Cette étude porte sur l'acquisition des prépositions françaises à et de par des locuteurs tchèques et sur l'influence que la langue maternelle peut avoir sur cette acquisition. Le tchèque et le français étant deux langues génétiquement et typologiquement différentes, les apprenants sont au cours de leur acquisition du français confrontés à maints problèmes, parmi lesquels l'emploi des prépositions. Le tchèque, en tant que langue flexionnelle, possède en effet un système de déclinaisons qui permet d'utiliser des constructions à complément direct (portant un cas morphologique spécifique) là où le français utilisera obligatoirement une préposition. En outre, le tchèque peut utiliser d'autres moyens morphosyntaxiques (adjectifs, propositions subordonnées, substantifs déverbaux) là où le français utilisera des constructions prépositionnelles. Il n'est donc pas possible d'attribuer un équivalent unique aux prépositions françaises comme à et de, qui apparaissent dans des environnements syntaxiques très variés.

notre étude, nous avons examiné les prépositions à et de dans les constructions à élément recteur verbal, nominal, adjectival et adverbial, dans lesquelles leur apport sémantique est plus ou moins difficile à cerner (sens spatial, directionnel, abstrait, co-désignation d'un bénéficiaire, d'une source, etc.). Nous avons construit deux tests portant sur ces constructions (test d'acceptabilité et test à trou) et nous les avons soumis à 52 apprenants tchèques du français (des étudiants universitaires). Ces tests nous ont permis d'identifier, d'une part, des constructions où les apprenants produisent peu ou pas d'erreurs, et, d'autre part, des constructions où les apprenants produisent des erreurs de manière systématique. En nous appuyant sur la comparaison entre le français et le tchèque, nous avons montré que l'acquisition de ces constructions peut s'expliquer par l'influence de la langue maternelle des apprenants, qu'il s'agisse d'un transfert positif dans le cas des constructions correctes (p. ex. préposition $z>$ préposition de, préposition $v>$ préposition $\grave{a}$, génitif $>d e$, datif $>\grave{a}$ ) ou de transfert négatif dans le cas des erreurs (prépositionod $>* d e$ au lieu de $a$, préposition $s>*^{*}$ avec au lieu de de). L'hypothèse supplémentaire selon laquelle l'apprentissage de certaines constructions (p.ex. verbales) dès le début du cursus permettrait de les fixer rapidement et définitivement n'a cependant été confirmée que partiellement (cf. la construction dire à qqn >*dire qqn). Par ailleurs, la variation de certaines constructions verbales (continuer à / continuer de) ne facilite pas leur fixation chez les apprenants.

Cette étude soulève d'autres questions qui n'ont pas pu y être abordées. Il serait par exemple intéressant de compléter nos résultats transversaux par une étude longitudinale, qui permettrait de montrer les stades développementaux de l'acquisition des prépositions à et de. Il serait également pertinent de s'interroger si les connaissances d'autres langues étrangères ont une influence sur l'acquisition de à et de en français. Enfin, nous envisageons, dans nos recherches ultérieures, d'exploiter 
les résultats de notre étude pour faire des propositions didactiques concrètes visant à améliorer l'acquisition de ces deux prépositions.

\section{BIBLIOGRAPHIE}

Cox, Thomas J. (1983). Teaching the Unteachable: Prepositional Complementizers in French.

The French Review, 57(2), 168-178.

De Groot, Annette M. B. \& KeIJZER, Rineke. (2000). What Is Hard to Learn Is Easy to Forget: The Roles of Word Concreteness, Cognate Status, and Word Frequency in Foreign Language Vocabulary Learning and Forgetting. Language Learning, 50, 1-56.

GAFFNEY, Caitlin (2015). L'acquisition L2 des compléments infinitifs français par des apprenants anglophones. Arborescences : revue d'études françaises, 5, 63-96.

HuoT, Hélène. (1981). Constructions infinitives du français. Le subordonnant de. Droz.

KelleRman, Eric \& SHARWOOD SMIth, Michael. (1986). Cross-Linguistic Influence in Second Language Acquisition. Pergamon Press.

KirjaVAinen, Minna, THeakston, Anna, Lieven, Elena \& Tomasello, Michael. (2009). 'I Want Hold Postman Pat': An Investigation into the Acquisition of Infinitival Marker 'to'. First Language, 29(3), 313-339.

LONSDALE, Deryle \& LE BRAS, Yvon. (2009). Routledge Frequency Dictionary of French: Core Vocabulary for Learners. Routledge.

MARQUE-PUCHEU, Christiane. (2008). La couleur des prépositions à et de. Langue française, 157, 74-105.

ODLIN, Terence. (1989). Language Transfer: Crosslinguistic Influence in Language Learning. Cambridge University Press.

ODLIN, Terence. (2003). Crosslinguistic Influence. Dans C. Doughty \& M. Long (dir.), Handbook of Second Language Acquisition (p. 436-486). Blackwell.

RIEGEL, Martin, PELLAT, Jean-Christophe \& Rioul, René. (2018). Grammaire méthodique du français (7 $7^{\mathrm{e}}$ éd.). Presses universitaires de France.

RUTHERFORD, William. (1983). Language Typology and Language Transfer. Dans S. M. Gass \& L. Selinker (dir.), Language Transfer in Language Learning (p. 358-370). Newbery House.

SELINKER, Larry. (1969). Language Transfer. General Linguistics, 9, 67-92.

SELINKER, Larry. (1972). Interlanguage. International Review of Applied Linguistics, 10, 209-241.

TowelL, Richard \& HAWKINS, Roger. (1994). Approaches to Second Language Acquisition. Multilingual Matters. 


\section{Manuels}

CHAHI, Fatiha et coll. (2015). Entre nous. Maison des langues.

GIRARDET, Jacky \& PÉCHEUR, Jacques. (2012). Écho junior A1-B1. CLE International / Sejer.

MÉRIEUX, Régine \& LoISEAU, Yves. (2004). Connexions - méthode de français - niveau 1. Éditions Didier.

PravdovÁ, Marie \& PRAVDA, Miroslav. (2007). Francouzština nejen pro samouky. LEDA.

TIBERT, Simone. (2003). Le français avec des jeux et des activités (Niveau pré-intermédiaire). ELI.

\section{NOTES}

1. Nous ne traitons pas des séquences de type beaucoup de comme des déterminants complexes. Nous pouvons voir lors de leur pronominalisation que c'est « de N » et non «beaucoup de » qui fonctionne comme une unité syntaxique : « il y avait beaucoup de gens sur la plage » > « il y en avait beaucoup ». Par ailleurs, les apprenants produisent des séquences dans lesquelles la forme de varie : *beaucoup des gens, *beaucoup du chocolat.

2. «Langue et littérature françaises » en combinaison avec «Langue et littérature anglaises » ou «Sciences humaines / lettres ».

3. Les sujets n'étaient pas assez nombreux (52) pour constituer plusieurs groupes dont les résultats seraient statistiquement significatifs.

4. Manuels utilisés notamment dans l'enseignement secondaire : Entre nous 1-2 (Chahi et coll., 2015), Écho junior A1-B1 (Girardet \& Pécheur, 2012), Connexions - méthode de français - niveau 1 (Mérieux \& Loiseau, 2004), Francouzština nejen pro samouky [Le français non seulement pour autodidactes] (Pravdová \& Pravda, 2007).

5. Dans les tableaux 5 à 8 , les réponses correctes sont indiquées en nombre d'occurrences sur nombre total et en pourcentage. Pour confirmer la différence significative des résultats obtenus dans chaque groupe d'un point de vue statistique, nous avons appliqué la variante Welch du test $\mathrm{T}$ de Student. Le test $\mathrm{T}$ confirme à chaque fois que le $\mathrm{G} 2$ a un meilleur niveau que le $\mathrm{G} 1$. Concrètement, pour toutes les expériences, la valeur du test $\mathrm{T}$ indique une probabilité plus petite que 0,05 pour que les résultats se présentent comme c'est le cas, alors que G1 et $\mathrm{G} 2$ ont mêmes capacités : $\mathrm{t}=0,0000339772($ Exp. $1: \grave{a}), \mathrm{t}=0,0003093064($ Exp. $1:$ de) $, \mathrm{t}=0,0250351787($ Exp. $1: \grave{a}$ ou de), $\mathrm{t}=0,0192744187$ (Exp. $2: \grave{a}), \mathrm{t}=0,0003030712($ Exp. $2: \mathrm{de}), \mathrm{t}=0,0005781177$ (Exp. $2: \grave{a}$ ou de). Le test $\mathrm{T}$ rejette ainsi l'hypothèse « les deux groupes ont le même niveau » en faveur de l'hypothèse «G2 a un meilleur niveau que G1». Nous tenons à remercier Christoph Kriegler, enseignantchercheur en mathématiques à l'UCA, de nous avoir aidé à effectuer les calculs statistiques.

\section{RÉSUMÉS}

Dans cette contribution, nous nous interrogeons sur l'acquisition des prépositions à et de en français par des locuteurs tchèques et sur le rôle positif ou négatif de la langue maternelle dans ce processus. Notre étude porte sur les prépositions à et de dans des constructions à élément recteur verbal, nominal, adjectival, et adverbial. Ces constructions ont été soumises à des 
apprenants tchèques du français (étudiants à l'université) sous forme de deux tests (test de grammaticalité, test à trou). Ces tests nous ont permis d'identifier les constructions où les apprenants produisent peu ou pas d'erreurs et celles où les apprenants produisent des erreurs de manière systématique. En nous appuyant sur la comparaison entre le français et le tchèque, nous avons également émis des hypothèses sur de possibles transferts du tchèque vers le français. Les résultats de nos tests rendent plausible aussi bien l'hypothèse de certains transferts positifs que celle de certains transferts négatifs.

This paper deals with the acquisition of the French prepositions $\grave{a}$ and de by Czech speakers and the positive or negative role of the native language in this process. Our study focuses on the prepositions $a$ and de in constructions containing verbal, nominal, adjectival and adverbial rector elements. These constructions were submitted to Czech university students of French using two tests (grammaticality judgment and gap-fill exercises). The tests enabled us to identify the constructions in which learners make few or no errors and those where learners systematically produce errors. We also formulated hypotheses about possible transfers from Czech into French, based on the comparison of both languages. The results of our tests make the hypothesis of some positive as well as some negative transfers plausible.

\section{INDEX}

Mots-clés : acquisition du français, prépositions à et de, constructions syntaxiques avec à et de, transfert positif et négatif

Keywords : acquisition of French, prepositions à and de, syntactic constructions with à and de, positive and negative transfers

\section{AUTEURS}

\section{HANA GRUET-SKRABALOVA}

Université Clermont Auvergne

hana.gruet-skrabalova@uca.fr

\section{IVA DEDKOVÁ}

Université d'Ostrava

iva.dedkova@osu.cz 